\title{
Genetics and Genomics of Infectious Diseases: advancing our understanding of host/pathogens and their interactions Ninghan Yang and Martin L Hibberd
}

\author{
Address: Infectious Diseases, Genome Institute of Singapore, 6o Biopolis Street, 138672 Singapore. \\ Correspondence: Martin L Hibberd. Email: hibberdml@gis.a-star.edu.sg
}

Published: 18 May 2009

Genome Medicine 2009, I:52 (doi: I0.1 I86/gm52)

The electronic version of this article is the complete one and can be

found online at http://genomemedicine.com/content/I/5/52

(C) 2009 BioMed Central Ltd

\begin{abstract}
A report on the Genetics and Genomics of Infectious Diseases conference, Singapore, 2I-24 March 2009.
\end{abstract}

\section{Introduction}

The Genetics and Genomics of Infectious Diseases conference (http://www.nature.com/natureconferences/ggid2009/ index.html) gathered researchers from diverse areas of expertise to further our understanding of infectious diseases, integrating knowledge of different aspects of pathogen and host biology to form a holistic picture. It was the first time that two major genetic societies, the American Society of Human Genetics and the Human Genome Organisation, have collaborated to bring together scientists interested in different but interdependent aspects of infectious diseases. The conference was also co-hosted by the Nature Publishing Group, with support from the World Health Organization and the Ministry of Health of Singapore. The meeting attracted more than 250 participants, and around 30 talks and over 40 posters were presented, with four workshops given by biotechnology companies.

The meeting was able to integrate the diverse fields of human responses to infectious disease, microbe discovery, epidemiology, drug discovery and others by focusing on high-throughput genomic technologies. It was remarkable how this provided a common language to all the participants and allowed deep understanding of areas outside each individual's usual expertise.

Some of the more novel insights came from the direct application of these technologies by groups that might not see themselves as traditional microbiologists. For example, Rotem Sorek (Weizmann Institute of Science, Rehovot, Israel) described a novel method of antimicrobial discovery, following their realization that cloning gaps in microbial sequencing undertakings may be due to genes that kill the cloning microorganism, Escherichia coli. It was fascinating to hear the innovative perspective given to a common phenomenon that is not given much attention in sequencing and that could potentially be turned to high-throughput screening for antimicrobials through microfluidics. Daniel Falush (University College, Dublin, Ireland) also gave an absorbing talk about using new-generation genomic technology and analytical methods such as sloppy painting (a novel approach for understanding patterns of admixture between populations) to describe gene flow in Helicobacter pylori in detail, revealing that their migration across continents is tied to that of humans, and thus where their selective pressures come from - fascinating insights into 'the private lives of bacteria'.

There was also a glimpse of the future of how the genomics approach might be applied more systematically to infectious diseases from Ulf Nehrbass (Institute Pasteur, Seoul, Korea). His group established high-content screening, which enables live visualization together with evaluation of the infection process, within high level bio-safety containment. Such methodologies are more typically used by companies, but this approach was shown to give biological insights and 
mechanisms, as well as to identify antimicrobial drugs and their targets.

A unique feature of the conference was a discussion session on how advanced genomic sciences can be integrated into the framework of public health and government policies to combat infectious diseases. With the rapid development of our understanding resulting from these approaches, implementation is going to be crucial, and several contributors made insightful remarks. However, it was the scene-setting talk from Tikki Pang (World Health Organization, Geneva, Switzerland) that captured the imagination. He reminded all present about how much work was required, as existing drugs are becoming less effective (with few new ones on the horizon) and new infectious agents are arriving constantly usually from those areas of the world with the least surveillance. In particular, diagnostics need to be better, cheaper and preferably in the hands of the doctors in primary health care settings. Another contributor in this session, Edison Liu (President of the Human Genome Organisation and Executive Director of the Genome Institute of Singapore), provided some possible solutions, highlighting that as microbiology, through genetic high-throughput technologies, is becoming an information science, sharing databases of information will become easier. Networks of countries and scientists could develop, and Liu pointed to the company Google, which has attempted to monitor infectious disease outbreaks using its internet-based system of information collation. Several overarching themes covered during the conference are discussed in more detail below.

\section{Virus epidemiology and evolution}

The microbiology field has been transformed by the ready availability of high-throughput sequencing, with influenza taken as a case study by many speakers. Edward C Holmes (Pennsylvania State University, University Park and the National Institutes of Health, Bethesda, USA) described several aspects, including the spatial and temporal dynamics of influenza (pointing out the difference between genetic and antigenic space), molecular viral evolution from a genomics viewpoint, and possible origins of amantadine-resistant strains. A striking realization from this was that new influenza strains arrive in the USA each year from South East Asia, rather than developing within the country. Sunetra Gupta (University of Oxford, UK), proposed an explanation for the rise of influenza pandemics, in which selection by the host immune system has a crucial role in determining pandemic influenza strains in a limited antigenic space. The optimism for this description of how new strains evolve was shared by Neil Ferguson (Imperial College London, UK), as his work predicted a drastic decrease in the spreading rate of drug-resistant strains as a result of highly stratified host population structures. Malik Peiris (University of Hong Kong, Hong Kong) also highlighted the need to re-examine the role of the host response in high-pathogenicity influenza strains and cautiously explored the possibility of using immunomodulation for therapy. It is likely that further research using cutting edge technology will continue to shed light and overturn widely held dogmas in this field.

\section{Role of indigenous microflora}

Another fast-moving field is the expanding research on gut microbiota, with speakers strongly putting forth the idea of the human as a 'superorganism', with indigenous microbiota having a complex and deep relationship with human biology. One of the highlights was delivered by Jacques Ravel (University of Maryland, College Park, USA), who proposed the idea that it is resilience that determines the composition of microbial communities, rather than a core microbiome as was the widely held opinion. Ravel's idea is strengthened by microbial survey data from human vaginal bacterial communities from women across different ethnic groups.

The importance of indigenous microbiota was further reinforced by Martin J Blaser (New York University Langone Medical Center, New York, USA), whose work covered the delicate balance between $H$. pylori and human physiology, with a decreased prevalence possibly linked to gastroesophageal disease and hormonal imbalances related to energy homeostasis. He suggested the 'disappearing microbiota' hypothesis (not to be confused with the hygiene hypothesis), in which dramatic changes in indigenous microbiota transmission and maintenance cause equally dramatic changes in human physiology. The research presented raised as many questions as it answered, and it would be interesting to see future research strengthening or altering the various hypotheses put forth or even forming relationships in unexpected ways.

\section{The genetics of the host response}

The human genetics field has been revolutionized by the recent introduction of the genome-wide association study (GWAS) approach. The infectious disease field has been applying this to a range of diseases, with GWAS covering tuberculosis (Fredrik Vannberg, Oxford University, Oxford, UK), human immunodeficiency virus (HIV; Stephen J O'Brien, National Cancer Institute, Rockville, USA), dengue (Cameron Simmons, Oxford University, Ho Chi Minh City, Vietnam), malaria (Yik Y Teo, Oxford University, Oxford and Cambridge University, Cambridge, UK) and leprosy (Laurent Abel, University Paris Descarte, Paris, France) presented at the meeting. Although this was certainly the cutting edge of the field, overall the presentations were perhaps more preliminary in the analysis stages, with many of the talks focusing on a discussion of methodological issues. A particularly detailed methodology came from O'Brien, who revealed a process for combining multiple analyses, both statistically and phenotypically, to obtain 
multiple lines of evidence for the involvement of a locus. Teo discussed the difficulties of using the standard GWAS approach in African populations, as the linkage disequilibrium structure is far more complex than in out-ofAfrica populations. However, if associations are found, African populations may be very helpful in narrowing down causal variants. He also highlighted the difficulties of identifying rare causal variants, using the sickle cell mutation as an example.

\section{Conclusions}

This highly invigorating and thought-provoking conference succeeded through a combination of excellent, first-rate speakers who are leaders in their fields, a highly focused theme (understanding host-pathogen interactions) tackled from the wide perspectives of human and pathogen genomes, and active discussion of the impact the work might have on those most affected by infectious disease. It would be exciting to hold another similar meeting for this fastmoving area in the near future.

\section{Abbreviations}

GWAS, genome-wide association study.

\section{Acknowledgements}

We thank all the speakers who presented their research during the meeting. 